\title{
Bastonetes Gram-negativos em úlceras venosas e implicações para o atendimento de enfermagem na atenção primária*
}

\author{
Gram-negative rods in venous ulcers and implications for primary care nursing care
}

Bacilos Gram-negativos en úlceras venosas, implicancias para la atención de enfermería en la atención primaria

Silvana de Lima Vieira dos Santos ${ }^{1}$, Marlene Andrade Martins ${ }^{2}$, Lara Stefânia Netto de Oliveira Leão Vasconcelos ${ }^{3}$, Ana Beatriz Mori Lima ${ }^{4}$, Suelen Gomes Malaquias ${ }^{5}$, Maria Márcia Bachion ${ }^{6}$

\footnotetext{
* Estudo originário da Tese de Doutorado intitulada: "Análise clínica e microbiológica de úlceras venosas de pacientes atendidos em Unidades Básicas de Saúde de Goiânia". Financiado pela Fundação de Amparo à Pesquisa no Estado de Goiás (FAPEG).

${ }^{1}$ Enfermeira, Doutora em Ciências da Saúde. Professora Adjunta da Faculdade de Enfermagem da Universidade Federal de Goiás (FEN/UFG). Goiânia, GO, Brasil. E-mail: silvanalvs@hotmail.com.

${ }^{2}$ Enfermeira, Doutora em Ciências da Saúde. Professora Adjunta do Departamento de Enfermagem da UFG - Campus Jataí (CAJ/UFG). Jataí, GO, Brasil. Email: marleniapower@hotmail.com.

${ }^{3}$ Biomédica, Doutora em Ciências da Saúde. Professora Adjunta do Instituto de Patologia e Saúde Pública (IPTSP) da UFG. Goiânia, GO, Brasil. E-mail: larastefania@yahoo.com.br.

${ }^{4}$ Biomédica, Doutora em Ciências da Saúde. Professora Adjunta do IPTSP/UFG. Goiânia, GO, Brasil. E-mail: anabeatrizmori@yahoo.com.br.

${ }^{5}$ Enfermeira, Mestre em Enfermagem. Discente do Programa de Pós-Graduação em Ciências da Saúde, nível Doutorado, da Faculdade de Medicina da UFG.

Professora Assistente do Departamento de Enfermagem da CAJ/UFG. Jataí, GO, Brasil. E-mail: sgmalaquias@gmail.com

${ }^{6}$ Enfermeira, Doutora em Enfermagem. Professora Titular da FEN/UFG. Goiânia, GO, Brasil. E-mail: mbachion@gmail.com
}

\section{RESUMO}

Estudo transversal descritivo em pacientes com úlceras venosas de difícil cicatrização, cujos objetivos foram identificar a prevalência de bastonetes Gram-negativos (BGN) em úlceras venosas com sinais clínicos de infeç̧ão; analisar o perfil de suscetibilidade destes e detectar a produção de ESBL, AmpC e metalo- $\beta$-lactamase. Nesta amostra, identificaram-se 69 pessoas. A coleta foi realizada mediante entrevista, exame clínico, registro fotográfico e swab das lesões. Os espécimes foram submetidos à avaliação laboratorial para análise microbiológica. Foram avaliadas 98 lesões, sendo isolados BGN em 74,5\%. Prevaleceram P. aeruginosa e Escherichia coli. Os micro-organismos foram sensíveis à maioria dos antimicrobianos. Em relação à resistência, destacaram-se tetraciclina e cefoxitina. ESBL não foi detectada, AmpC foi verificada em 37,5\% no grupo CESP e a metalo- $\beta$-lactamase em $8,0 \%$ das $P$. aeruginosa. $O$ estudo indica a necessidade de vigilância microbiológica para os pacientes com úlceras venosas com processo cicatricial de difícil evolução.

Descritores: Úlcera Varicosa; Análise Microbiológica; Atenção Primária à Saúde; Cuidados de Enfermagem.

\section{ABSTRACT}

A descriptive cross-sectional study in patients with hard-to-heal venous ulcers, whose objective is to identify the prevalence of Gram-negative rods (GNR) in venous ulcers presenting clinical signs of infection; analyze the susceptibility profile of such ulcers and detect ESBL, AmpC and metallo- $\beta$-lactamase production. We identified 69 individuals. The samples were gathered by means of an interview, clinical exam, photographic record and a swab of the lesions. Specimens were submitted to laboratorial evaluation for microbiological analysis. A total of 98 lesions were evaluated, of which BGN presented in $74.5 \%$. There was a prevalence of $P$. aeruginosa and Escherichia coli. The mircoorganisms were sensitive to most antimicrobials. In terms of resistance, tetracycline and cefoxitine were discarded. ESBL was not detected; AmpC was verified in $37.5 \%$ of the CESP group and metalo- $\beta$-lactamase in $8.0 \%$ of $P$. aeruginosa. Our study points to the need for microbiological vigilance of patients with hard-to-heal venous ulcers.

Descriptors: Varicose Ulcer; Microbiological Analysis; Primary Health Care; Nursing Care.

\section{RESUMEN}

Estudio transversal descriptivo en pacientes con úlceras venosas de difícil cicatrización, objetivando identificar la prevalencia de bacilos Gram-negativos (BGN) en ulceras venosas con signos clínicos de infección; analizar su perfil de susceptibilidad y detectar la producción de ESBL, AmpC y metalo- $\beta$-lactamasa. Fueron identificadas 69 personas. Datos recolectados mediante entrevista, examen clínico, registro fotográfico y swab de lesiones. Los especímenes fueron sometidos a evaluación laboratorial para análisis microbiológico. Fueron evaluadas 98 lesiones, aislándose BGN en $74,5 \%$. Prevalecieron $P$. aeruginosa y Escherichia coli. Los microorganismos fueron sensibles a la mayoría de antimicrobianos. Respecto a resistencia, se destacó la misma respecto de tetraciclina y cefoxitina. No se detectó ESBL, se verificó AmpC en $37,5 \%$ en el grupo CESP y la metalo- $\beta$ lactamasa en $8,0 \%$ de las $P$. aeruginosa. El estudio indica necesidad de vigilancia microbiológica para pacientes con úlceras venosas con proceso cicatricial de difícil evolución.

Descriptores: Úlcera Varicosa; Análisis Microbiológico; Atención Primaria de Salud; Atención de Enfermería. 


\section{INTRODUÇÃO}

Entre os fatores que dificultam a cicatrização das úlceras vasculares está a infecção que geralmente ocorre devido à exposição de longa duração das áreas com solução de continuidade ao meio ambiente, associada a condições precárias de saúde do hospedeiro ${ }^{(1)}$.

Geralmente, entre os micro-organismos presentes nos processos infecciosos das úlceras vasculares encontram-se os Gram-positivos, principalmente Staphylococcus aureus, e os bastonetes Gram-negativos (BGN). Neste último grupo de micro-organismos destacam-se as enterobactérias como Escherichia coli e Proteus mirabilli, e os bastonetes Gram-negativos Nãofermentadores (BGNNF) como Pseudomonas aeruginosa ${ }^{(2-3)}$.

Os BGN têm preocupado a comunidade científica de modo mais acentuado nos últimos anos, devido ao perfil de resistência aos antimicrobianos ${ }^{(4)}$. Entre os mecanismos de resistência dos BGN (enterobactérias e BGNNF), a produção de enzimas $\beta$-lactamases tem recebido especial destaque devido à sua emergência, tanto na comunidade quanto nos serviços de saúde ${ }^{(5-7)}$.

As principais $\beta$-lactamases produzidas por BGN são as enzimas ESBL ( $\beta$-lactamase de espectro ampliado), AmpC e a MBL (metalo- $\beta$-lactamase) ${ }^{(5)}$. As ESBL são enzimas não induzíveis e transmitidas por meio de plasmídeos. Os micro-organismos produtores apresentam resistência aos beta-lactâmicos de amplo espectro como as cefalosporinas de terceira geração (ceftazidima, cefotaxima e ceftriaxona) e os monobactâmicos (aztreonam) ${ }^{(5)}$. Com a realização do antibiograma tradicional, não é possível a detecção de cepas produtoras de ESBL, sendo necessária a realização de testes adicionais ${ }^{(5)}$. Segundo protocolo do Clinical and Laboratory Standars Institute-CLSI $2009^{(8)}$, esta enzima deve ser pesquisa para Klebsiella pneumoniae, $K$. oxytoca e E. coli.

A enzima AmpC pode ser produzida de modo constitutivo (E. coli, K. pneumoniae, K. oxytoca) ou induzido por antimicrobianos (Citrobacter freundii, Enterobacter spp., Serratia spp., Providencia spp., Moragnella morganii e $P$. aeruginosa $)^{(9)}$. A presença do gene de indução está relacionada a resultado de falsa sensibilidade na realização dos testes de suscetibilidade aos antimicrobianos ${ }^{(10)}$. Uma vez instituída a terapêutica, o mesmo micro-organismo pode expressar resistência, comprometendo a qualidade da assistência prestada ao indivíduo(10). Esta enzima é capaz de hidrolisar penicilinas, cefalosporinas e monobactâmicos ${ }^{(11)}$.
A MBL, produzida por vários BGN, especialmente $P$. aeruginosa, caracteriza-se por necessitar de zinco $(\mathrm{Zn++})$, como cofator para a reação de hidrólise do anel beta-lactâmico (o qual confere atividade bactericida), sendo detectada por meio de testes fenotípicos com o auxílio de agentes quelantes, como os ácidos etilenodiaminotetracético (EDTA) e 2-mercaptopropiônico $(\text { MPA })^{(12)}$

É de grande importância clínica e epidemiológica a $M B L$, pois atua sobre todos os $\beta$-lactâmicos de largo espectro comercialmente disponíveis incluindo os carbapenens. Porém esta enzima não é capaz de hidrolisar o monobactâmico (aztreonam) ${ }^{(13)}$.

São conhecidas seis subclasses de MBL adquiridas: imipenemase (IMP), Verona imipenemase (VIM), São Paulo metalo-beta-lactamase (SPM-1), German imipenemase (GIM-1), Seoul imipenemase (SIM-1) e Australian imipenemase (AIM), sendo as frequentes no Brasil SPM, IMP e VIM ${ }^{(14)}$. Em relação à MBL tipo SPM-1, esta não atua sobre o aztreonam e sobre o fármaco ácido clavulânico, o qual é utilizado em associação com a ticarcilina ${ }^{(15)}$. A detecção de micro-organismos produtores de MBL é uma ferramenta auxiliar no controle de infecções em feridas e orientação adequada da terapêutica ${ }^{(16)}$.

Uma vez identificados estes fenótipos de resistência entre BGN isolados de feridas compreende-se que a instituição dos antimicrobianos beta-lactâmicos está comprometida, a excessão das cefaminas $^{(5)}$. Esta condição sinaliza a dificuldade de instituição de uma terapêutica adequada e consequentemente o restabelecimento das úlceras venosas.

A decisão clínica de considerar ou não uma úlcera infectada está pautada, inicialmente, em sinais como presença de tecido de granulação friável, de coloração cor de tijolo, com surgimento abrupto de manchas acastanhadas, celulite, falta de progresso na cicatrização, mesmo com aplicação de terapia compressiva, entre outros ${ }^{(17)}$. Culturas de microorganismos a priori para todas as lesões são desaconselhadas, pelo alto custo ${ }^{(18-19)}$ e também pelo fato da simples existência dos micro-organismos não determinar por si a deterioração da lesão, uma vez que a infecção depende da virulência do micro-organismo, da resistência ${ }^{(19)}$ do hospedeiro e da exposição(2).

No atendimento às pessoas com úlceras venosas na atenção primária à saúde, a Enfermagem manipula as lesões durante a realização dos curativos e deve decidir sobre as coberturas a serem utilizadas em cada situação. Quando detecta, na sua avaliação clínica, sinais e 
sintomas de infecção, deve instituir produtos com ação antimicrobiana como a prata nanocristalina e o cadexômero iodine ${ }^{(19-20)}$. Outras alternativas são papaína a $10 \%$ e alginato de cálcio(21).

No caso de não se conseguir reverter a situação e a úlcera mostrar-se refratária a estas medidas, condutas envolvendo outros profissionais são indicadas, incluindo a coleta de material para análise microbiológica ${ }^{(17)}$. Enquanto se aguarda o resultado do antibiograma é instituído um antibiótico de largo espectro ${ }^{(8)}$, contudo para essa escolha é necessário conhecer o perfil dos micro-organismos usualmente encontrados. Em todo esse processo a enfermagem monitora os resultados da terapêutica instituída e participa das medidas de controle de infecção. $\mathrm{Na}$ constatação de micro-organismos resistentes devem ser instituídas precauções de contato $^{(7)}$ e a orientação necessária deve ser prestada ao paciente.

A existência de poucos dados epidemiológicos acerca dos agentes etiológicos associados às infecções em úlceras vasculares e dos seus mecanismos de resistência dificulta a formulação e adoção de políticas adequadas de controle de infecções e terapia empírica antimicrobiana de assistência à saúde nessa área específica de atendimento.

Os objetivos do estudo foram identificar a prevalência de bastonetes Gram-negativos (enterobactérias e não-fermentadores) em úlceras venosas com sinais clínicos de infecção; analisar o perfil de suscetibilidade dos isolados; e detectar a produção de $\beta$-lactamases tipo ESBL, AmpC, e metalo- $\beta$-lactamase.

\section{MATERIAL E MÉTODOS}

Este é um estudo transversal, aninhado num projeto, intitulado "Avaliação de úlceras venosas de estase no contexto do atendimento ambulatorial na rede municipal de saúde de Goiânia: ampliando as perspectivas", aprovado pelo Comitê de Ética em Pesquisa da Universidade Federal de Goiás (protocolo 041/2009). Foi realizado em 49 salas de curativos, com atendimento a pessoas com feridas crônicas, na rede de atenção primária a saúde, no município de Goiânia (Goiás, Brasil).

No processo de recrutamento dos sujeitos, os pesquisadores compareceram às unidades de saúde que atendiam pessoas com feridas crônicas e, ao identificar pessoas com presença de curativos nos membros inferiores, iniciavam a abordagem, apresentando-se e investigando o motivo da procura pelo atendimento na sala de curativos. Caso o paciente relatasse a presença de úlceras nos membros inferiores, informava-se sobre a pesquisa e solicitava-se autorização para observação da realização do curativo, com a finalidade de verificação de possível elegibilidade para o estudo, ou seja: idade $\geq 18$ anos e presença de úlcera venosa com ou sem complicação arterial. Verificada a elegibilidade, passavase a verificar os critérios de inclusão na pesquisa: presença de um dos sinais de infecção descrito pela European Wound Management Association (EWMA) ${ }^{(18)}$. Para aqueles que atenderam a esse critério formulou-se o convite para participação no estudo. No caso de aceite, era oferecido o termo de consentimento livre e esclarecido (TCLE), para assinatura.

Para a coleta de dados foi aplicado um roteiro de entrevista contendo dados sociodemográficos e características clínicas das lesões. Para avaliação da presença de sinais e sintomas clínicos de infecção, foi utilizado o protocolo descrito pela EWMA ${ }^{(18)}$.

A coleta dos espécimes se deu conforme a técnica de Levine $^{(22)}$, na qual gira-se o swab durante cinco segundos em seu próprio eixo, em um único ponto do leito da ferida (tecido de granulação viável) utilizando-se swab alginatado esterilizado e a seguir, inocula-se o mesmo em meio para transporte de amostras biológicas (meio de Stuart). O material foi então transportado para o laboratório em temperatura ambiente e processado dentro de um prazo de até 12 horas após a coleta ${ }^{(9)}$. Em todos os testes realizados, foram empregadas cepas padrão (Escherichia coli ATCC 25922, Pseudomonas aeruginosa ATCC 27853) da American Type Culture Collection, como controle de qualidade.

As análises microbiológicas para isolamento e identificação seguiram técnicas recomendadas por Oplustil $^{(9)}$ e Koneman ${ }^{(10)}$. O isolamento primário dos BGN foi realizado inoculando o espécime clínico em ágar MacConkey, seguida de incubação a $35^{\circ} \mathrm{C}$ por $18-24$ horas. Em seguida, foram realizados testes de triagem (cultivo em ágar Kligler Ferro e prova de detecção de citocromo oxidase) e provas bioquímicas de identificação.

Para a identificação das enterobactérias foram realizadas as seguintes provas: produção de indol, motilidade, utilização de citrato, produção de urease, produção de fenilalanina-desaminase, vermelho de metila, produção de sulfeto de hidrogênio, descarboxilação da lisina, arginina e ornitina.

Para a identificação dos BGNNF foram empregadas as provas de descarboxilação da arginina e lisina, crescimento a $42^{\circ} \mathrm{C}$, produção de pigmentos característicos, suscetibilidade à polimixina $B$, produção de urease, utilização do citrato e motilidade. 
O perfil de suscetibilidade antimicrobiana (antibiograma) dos BGN foi determinado pelo método de disco-difusão, segundo recomendação do Clinical and Laboratory Standards Institute ${ }^{(8)}$ A escolha dos discos de antimicrobianos foi realizada com base no perfil dos micro-organismos e do sítio anatômico investigado (úlceras venosas) $^{(23)}$.

Os antimicrobianos avaliados para enterobactérias foram: amoxacilina/ácido clavulânico (20/10 $\mu \mathrm{g})$, cefpodoxima $(10 \mu \mathrm{g})$, ceftriaxona $(30 \mu \mathrm{g})$, tetraciclina $(30 \mu \mathrm{g})$, aztreonam $(20 / 10 \mu \mathrm{g})$, cefepima $(30 \mu \mathrm{g})$, cefotaxima $(30 \mu \mathrm{g})$, cefoxitina $(30 \mu \mathrm{g})$, ceftazidima $(30 \mu \mathrm{g})$, ciprofloxacina $(5 \mu \mathrm{g})$, gentamicina $(10 \mu \mathrm{g})$, imipenem $(10 \mu \mathrm{g})$, levofloxacina $(5 \mu \mathrm{g})$, meropenem $(10 \mu \mathrm{g})$, piperacilina/tazobactam (100/10 $\mu \mathrm{g})$, trimetropim/sulfametoxazol (1.25/23.75 $\mu \mathrm{g})$.

Para os BGNNF foram empregados os seguintes antimicrobianos: amicacina $(30 \mu \mathrm{g})$, polimixina B (300 U), $(100 / 10 \mu \mathrm{g})$, aztreonam $(20 / 10 \mu \mathrm{g})$, cefepima $(30 \mu \mathrm{g})$, cefotaxima $(30 \mu \mathrm{g})$, cefoxitina $(30 \mu \mathrm{g})$, ceftazidima $(30 \mu \mathrm{g})$, ciprofloxacina $(5 \mu \mathrm{g})$, gentamicina $(10 \mu \mathrm{g})$, imipenem $(10 \mu \mathrm{g})$, levofloxacina $(5 \mu \mathrm{g})$, meropenem $(10 \mu \mathrm{g})$, piperacilina/tazobactam (100/10 $\mu \mathrm{g})$, trimetropim/sulfametoxazol (1.25/23.75 $\mu \mathrm{g})$.

Em relação à deteç̧ão dos fenótipos de resistência, para enterobactérias foram pesquisadas a produção de $\beta$ lactamases tipo ESBL e AmpC, Enquanto que para os BGNNF foram pesquisadas a produção de AmpC e metalo-beta-lactamase.

Para ESBL foi realizado o teste de (discoaproximação) $^{(8)}$, empregando-se as cepas padrão Klebsiella pneumoniae ATCC 700603 e Escherichia coli ATCC 25922. A detecção da enzima AmpC foi realizada por disco-aproximação ou double-disc sinergism segundo recomendações ${ }^{(5,9)}$, utilizando-se como controle as cepas padrão Escherichia coli ATCC 25922, Pseudomonas aeruginosa ATCC 27853. Já a produção de metalo-betalactamase foi detectada pelo teste de disco aproximação descrito por Arakawa (2000) ${ }^{(12)}$ sendo as cepas padrão Escherichia coli ATCC 25922 e Pseudomonas aeruginosa ATCC 27853.

Todos os resultados da análise microbiológica foram entregues aos participantes e informadas ao enfermeiro responsável pelas respectivas salas, para encaminhamento dos pacientes ao médico responsável e prestadas as orientações de precaução de contato.

Os dados foram analisados mediante procedimentos de estatística descritiva (frequências absoluta e relativa, moda e mediana), utilizando-se o Statistic Package for Social Sciences for Windows - SPSS ${ }^{\circledR}$ (versão 17.0).

\section{RESULTADOS}

Foram avaliados 69 pacientes, os quais apresentaram de uma a cinco lesões, sendo a moda igual a uma lesão e mediana de duas lesões, totalizando 98 úlceras. Predominou entre os participantes o gênero masculino $(58,0 \%)$, com idade que variou de 28 a 93 anos, sendo a mediana de 62 anos. O tempo de duração das lesões referido, foi de dois meses a 66 anos, sendo a mediana de 27 meses (2,25 anos) e moda de 60 meses (cinco anos).

Com relação à análise microbiológica, verificou-se que em $74,5 \%$ das lesões houve crescimento de BGN, perfazendo um total de 91 BGN isolados. Foram identificadas bastonetes da família Enterobateriaceae $(53,8 \%)$ e do grupo dos bastonetes Gram-negativos não fermentadores $(46,2 \%)$ (Tabela 1$)$.

Tabela 1. Distribuição dos bastonetes Gram-negativos identificados $(n=91)$ em úlceras venosas de pacientes atendidos em salas de curativos das Unidades Básicas de Saúde em Goiânia, GO, Brasil, 2010.

\begin{tabular}{|c|c|c|}
\hline Micro-organismos & $\mathbf{n}$ & $\%$ \\
\hline Bastonetes Gram-negativos não fermentadores & 42 & 46,2 \\
\hline Pseudomonas aeruginosa & 25 & 59,5 \\
\hline Pseudomonas sp. & 03 & 7,1 \\
\hline Outros BGNNF & 14 & 33,4 \\
\hline Enterobateriaceae & 49 & 53,8 \\
\hline Citrobacter freundii & 02 & 4,1 \\
\hline Citrobacter $s p$. & 01 & 2,0 \\
\hline Enterobacter aerogenes & 06 & 12,2 \\
\hline Enterobacter gergovia & 01 & 2,0 \\
\hline Escherichia coli & 12 & 24,5 \\
\hline Klebsiella oxytoca & 04 & 8,2 \\
\hline Klebsiella Pneumoniae & 02 & 4,1 \\
\hline Morganela morganii & 02 & 4,1 \\
\hline Pantoea aglomerans & 06 & 12,2 \\
\hline Proteus mirabillis & 06 & 12,2 \\
\hline Proteus vulgaris & 04 & 8,2 \\
\hline Providencia rettgeri & 03 & 6,2 \\
\hline
\end{tabular}


Entre as enterobactérias, prevaleceu a espécie Escherichia coli $(24,5 \%)$, seguida de Enterobacter aerogenes, Pantoea aglomerans e Proteus mirabillis $12,2 \%$ cada um conforme mostrado na Tabela 1.

Identificou-se ainda a presença de Klebsiella oxytoca em quatro lesões $8,2 \%$ e Klebsiella pneumoniae em duas lesões 4,1\% (Tabela 1).

Em relação aos BGNNF (bastonetes Gram-negartivos não fermentadores), destacou-se a prevalência do gênero Pseudomonas $66,6 \%$ e em especial a espécie $P$. aeruginosa $(59,5 \%)$, presente em $25,5 \%$ das amostras clínicas obtidas das feridas (Tabela 1 ).

Quanto ao perfil de suscetibilidade das enterobactérias, destacaram-se a resistência à tetraciclina $(38,8 \%)$, à amoxacilina-ácido clavulânico $(26,5 \%)$ e a sulfametoxazol-trimetroprim (24,5\%). As enterobactérias foram $100 \%$ sensíveis a oito dos antimicrobianos avaliados, incluindo os carbapenens. As quinolonas também apresentaram atividade para a maioria destes isolados (Tabela 2).

Tabela 2. Perfil de suscetibilidade de Enterobactérias $(n=49)$ isoladas de úlceras venosas de pacientes atendidos em salas de curativos das Unidades Básicas de Saúde de Goiânia, GO, Brasil, 2010.

\begin{tabular}{|c|c|c|c|c|c|c|}
\hline \multirow{2}{*}{ Antibimicrobianos } & \multicolumn{2}{|c|}{$\mathbf{R} *$} & \multicolumn{2}{|c|}{ S* } & \multicolumn{2}{|c|}{$\mathbf{I} *$} \\
\hline & $\mathbf{n}$ & $\%$ & $\mathbf{n}$ & $\%$ & $\mathbf{n}$ & $\%$ \\
\hline Amoxacilina/ácido clavulânico & 13 & 26,5 & 34 & 69,4 & 02 & 4,1 \\
\hline Aztreonam & - & - & 49 & 100,0 & - & - \\
\hline Cefepima & - & - & 49 & 100,0 & - & - \\
\hline Cefotaxima & - & - & 49 & 100,0 & - & - \\
\hline Cefoxitina & 07 & 14,3 & 42 & 85,7 & - & - \\
\hline Cefpodoxima & - & - & 49 & 100,0 & - & - \\
\hline Ceftazidima & - & - & 49 & 100,0 & - & - \\
\hline Ceftriaxona & - & - & 49 & 100,0 & - & - \\
\hline Ciprofloxacina & 06 & 12,2 & 43 & 87,7 & - & - \\
\hline Gentamicina & 03 & 6,1 & 46 & 93,8 & - & - \\
\hline Imipenem & - & - & 49 & 100,0 & - & - \\
\hline Levofloxacina & 06 & 12,2 & 42 & 85,7 & 01 & 2,0 \\
\hline Meropenem & - & - & 49 & 100,0 & - & - \\
\hline Piperacilina/tazobactam & - & - & 48 & 97,9 & 01 & 2,0 \\
\hline Tetraciclina & 19 & 38,8 & 28 & 57,1 & 02 & 4,1 \\
\hline Trimetoprim-sulfametoxazol & 12 & 24,5 & 34 & 69,4 & 03 & 6,1 \\
\hline
\end{tabular}

* $\mathrm{R}$ - resistente, $\mathrm{S}$ - sensível, I- intermediário

Os BGNNF apresentaram sensibilidade para vários dos antimicrobianos, incluindo beta-lactâmicos como meropenem, ceftazima e cefepime. Entre as $P$. aeruginosa, a maior resistência foi observada para cefoxitina $(100 \%)$ e sulfametoxazol-trimetroprim $(80,0 \%)$. As outras espécies de Pseudomonas sp. e os demais BGNNF apresentaram perfil de suscetibilidade semelhante.

As enterobactérias Escherichia coli, Klebsiella pneumonie, Klebsiella oxytoca e Proteus mirabillis isolados neste estudo foram submetidas à detecção do fenótipo de resistência ESBL, apresentando resultados negativos. Em relação à produção de enzima AmpC, $30,0 \%$ dos micro-organismos do grupo CESP e $100 \%$ das $P$. aeruginosa foram resistentes a cefoxitina. Os demais micro-organismos do grupo CESP (70,0\%) sensíveis à cefoxitina foram submetidos ao teste confirmatório e $37,5 \%$ apresentaram resultado positivo.

Quanto à detecção de metalo-beta-lactamase, $23,8 \%$ do BGNNF em geral apresentaram sensibilidade reduzida ao imipenem, meropenem ou ceftazidima. Ao serem submetidos ao teste confirmatório, observou-se que $8,0 \%$ das $P$. aeruginosa foram positivas para o mesmo.

\section{DISCUSSÃO}

Os BGN identificados nas úlceras venosas nesta pesquisa corroboram os achados de outros estudos que encontraram resultados semelhantes, em lesões crônicas de pele de um modo geral ${ }^{(2-3)}$. Contudo, a prevalência encontrada de BGN em 74,5\% das lesões superou os achados reportados na literatura, que variam de 47,0 a $60,0 \%$ para úlceras venosas ${ }^{(2,24)}$.

Os isolados identificados configuram em microorganismos que não fazem parte da microbiota residente da pele, e muitos deles são provenientes do intestino humano. Entre eles, E.coli, por exemplo, tem impacto clínico severo para os pacientes, uma vez que pode levar à amputações de membros ${ }^{(2)}$.

K. pneumoniae e a $K$. oxytoca também não são próprias da microbiota residente da pele. Estas podem ser isoladas de outros sítios humanos e também de fontes ambientais, especialmente a nosocomial ${ }^{(6)}$. Possui vários fatores de virulência, incluindo a produção de beta-lactamases e também a capacidade de formar 
biofilmes $^{(6)}$, o que interfere no processo de restabelecimento da lesão, e uma vez identificados os fenótipos de resistência as possibilidades terapêuticas são restritas ${ }^{(4)}$.

A identificação de Pseudomonas spp. nas feridas traz implicações para o prognóstico destas. Estudo realizado para determinar fatores associados ao retardo do processo de cicatrização de úlceras de perna verificou associação entre a presença de Pseudomonas e não restabelecimento destas lesões ${ }^{(24)}$.

Os isolados deste estudo têm tido destaque na literatura não só por interferirem no processo de cicatrização das úlceras ${ }^{(2)}$, mas também por apresentarem mecanismos de resistência aos antimicrobianos ${ }^{(13)}$, interferindo de maneira substancial na resposta terapêutica dos sujeitos acometidos por esses agentes patogênicos. Estudo ${ }^{(19)}$ recomenda que a prescrição de antimicrobianos sistêmico seja prescrito na vigência de sinais clínicos de infecção e não na presença de colonização.

No que se refere ao perfil de suscetibilidade os isolados foram sensíveis a vários antimicrobianos. Embora as enterobactérias tenham apresentado baixas taxas de resistência à tetraciclina e à amoxacilina-ácido clavulânico, este fato merece destaque, pois tratam-se de antimicrobianos de espectro ampliado ${ }^{(12)}$, sinalizando a necessidade de vigilância.

A resistência à cefoxitina detectada por este estudo (antibiograma) observada para alguns membros do grupo CESP e $P$. aeruginosa pode estar associada à hiperprodução de enzima AmpC por cepas mutantes, caracterizando a produção contínua desta enzima. Enquanto que para os micro-organismos do grupo CESP sensíveis à cefoxitina (antibiograma) e positivos para o teste de disco-aproximação a produção de AmpC irá se expressar durante a terapia com algum antimicrobiano indutor, caracterizando um mecanismo induzível. Este tipo de fenótipo é ainda mais preocupante por estar diretamente associado a falhas terapêuticas durante o tratamento com penicilinas e cefalosporinas de segunda e terceira geração(5).

Nesse contexto, mesmo com a suposta terapêutica adequada, com base no antibiograma, seu resultado deve ser interpretado levando-se em consideração o tipo de micro-organismo, sua patogenicidade e mecanismo de resistência ${ }^{(22)}$. É necessário que o enfermeiro mantenha a vigilância em relação aos sinais de infecção e evolução da lesão, pois a resistência induzida pode tornar ineficaz a ação do antimicrobiano em uso.
Apesar do baixo percentual de produção de MBL pelos BGNNF investigados, este dado merece destaque, pois a população em estudo encontra-se em ambiente ambulatorial e, geralmente, este fenótipo de resistência é identificado em isolados de infecções nosocomiais em unidades de alta complexidade ${ }^{(4,13)}$. Estudos ${ }^{(4,13)}$ têm identificado prevalência de MBL em isolados de unidades de alta complexidade entre 33 a 100\%. Já na atenção primária tem sido identificado em 50,0\% das enterobacteriaceas ${ }^{(25)}$.

Embora neste estudo tenha sido identificada 8,0\% de MBL nos isolados deve-se ter vigilância, pois estes pacientes podem vir a ter prejuízos referentes ao tratamento e restabelecimento de suas lesões, bem como a disseminação de micro-organismos com estes determinantes.

Em investigação para verificar a epidemiologia clínica, intervenções de controle de infecção e de vigilância ambiental de uma unidade de queimados foram detectas Enterobacteriaceas em pacientes e ambiência de quartos privativos com positividade para MBL e associação entre a contaminação ambiental e posterior colonização dos pacientes ${ }^{(16)}$.

Quanto a detecção de cepas com fenótipos de resistência, sugere-se que, os serviços de análises microbiológicas realizem testes de triagem fenotípica e confirmatórios $^{(17)}$ para a detecção correta de mecanismos de resistência de relevância clínica e epidemiológica, subsidiando a escolha da melhor conduta terapêutica a ser tomada pela equipe que assiste este paciente nas salas de curativos.

A escolha pela melhor conduta mediante análises microbiológicas, para tratamento das lesões pelo profissional da enfermagem repercutirá na assistência à saúde desses sujeitos, bem como para a instituição, pois mediante a identificação de micro-organismos multirresistentes além de ser instituído produtos ${ }^{(2,18)} \mathrm{com}$ atividade bactericidas e/ou bacteriostática para tratamento das úlceras venosas de difícil cicatrização, é recomendada a notificação aos serviços de controle de infecção (SCIH) das unidades de saúde, além de várias outras medidas de significante importância(7) como: "precaução de contato conjuntamente com as precauções padrão para todos os profissionais de saúde, visitantes e acompanhantes; fortalecer a política institucional de uso racional de antimicrobianos; estimular a higienização das mãos; avaliar a necessidade de implementar medidas de coorte em relação a profissionais de saúde e pacientes; avaliar a necessidade de implantar coleta de culturas de vigilância, de acordo 
com o perfil epidemiológico da instituição; enfatizar as medidas gerais de higiene do ambiente; aplicar, durante o transporte intrainstitucional e interinstitucional, as medidas de precauções de contato em adição às precauções-padrão para os profissionais que entram em contato direto com o paciente, incluindo o reforço nas medidas de higiene do ambiente; e comunicar, no caso de transferência intrainstitucional e interinstitucional, se o paciente é infectado ou colonizado por microrganismos multirresistentes", atividades que em sua maioria ficam sob responsabilidade do enfermeiro controlador de infecções relacionadas a assistência a saúde.

As recomendações brasileiras $^{(7)}$ de prevenção da disseminação de micro-organismos multirresistentes são para os estabelecimentos de saúde em geral, inclusive as unidades de atenção primária. Porém não há clareza de como fazer o manejo dos pacientes com úlceras venosas com micro-organismos multirresistentes na atenção primária, sendo necessário discutir as políticas públicas nesse sentido, uma vez que as medidas de proteção, prevenção e controle não se restringem apenas aos profissionais da área de saúde.

A identificação de cepas com os perfis de resistência pode ser indicativa de disseminação entre unidades, pois grande parte dos sujeitos do estudo se encontrava em acompanhamento especializado em unidades de alta complexidade e de referência no tratamento de pacientes com úlceras venosas. Uma maneira de confirmar a disseminação de clones interinstitucionais seria com a realização de testes genotípicos.

\section{CONCLUSÃO}

Os isolados mais prevalentes foram a $P$. aeruginosa seguida de $E$. coli. Os resultados apresentados evidenciaram que para as enterobactérias houve uma elevada prevalência de sensibilidade aos antimicrobianos: Aztreonam, Cefepima, Cefotaxima, Cefoxitina Cefpodoxima, Ceftazidima, Ceftriaxona, Ciprofloxacina, Gentamicina, Imipenem, Levofloxacina, Meropenem, e Piperacilina/tazobactam.

Verificou-se elevada prevalência de resistência a cefoxitina e sulfametoxazol-trimetroprim para a $P$. aeruginosa. Foi identificada baixa prevalência de fenótipos de resistência antimicrobiana para as Betalactamases AmpC e Metalo- $\beta$-lactamase aos antimicrobianos usualmente empregados na prática clínica, reforçando a necessidade do teste confirmatório. Apesar de ter sido observada uma baixa prevalência de produção destes fenótipos, são de grande relevância clínica para o contexto da atenção primária, especialmente no que se refere a produção de MBL.

Entre as implicações dos resultados para a prática clínica de enfermagem no atendimento à população com úlceras venosas, destaca-se a importância de medidas apropriadas de prevenção e controle de infecções, uma vez que as ações de limpeza e desinfecção da sala de curativos, do mobiliário, dos instrumentais podem ser influenciadas pela enfermagem, bem como a adequada lavagem de mãos, uso de equipamentos de proteção individual no atendimento aos usuários nesse local.

Quando não for possível dispor de salas de curativos nas unidades de saúde separadas para o tratamento de feridas limpas e de feridas infectadas, é fundamental a organização do agendamento dos usuários, de modo que aqueles que apresentam processo infeccioso nas lesões sejam atendidos ao final do turno de funcionamento da sala, com a necessária desinfeção entre os atendimentos.

Aumenta também a responsabilidade da enfermagem na avaliação de sinais clínicos

de infecção nas úlceras, a instituição de coberturas apropriadas e o monitoramento da evolução da cicatrização, uma vez que, na falta de resultados favoráveis, rapidamente deve-se mobilizar a equipe multiprofissional para as providências e encaminhamentos necessários, com vistas à provisão de terapêutica mais adequada.

Deve-se lembrar ainda que, em todo esse processo, a enfermagem deve realizar ações educativas junto aos usuários, não só para aumentar a capacidade dos mesmos para o autocuidado como para favorecer o seu empoderamento como cliente dos serviços

de saúde, os quais têm o dever de prestar atendimento de qualidade, o que inclui a segurança e resolubilidade dos serviços.

Para os gestores de saúde os resultados se colocam como um alerta para a necessidade de garantir adequadas condições de limpeza e funcionamento das salas de curativos, incluindo recursos humanos e insumos necessários, com estabelecimento de protocolos e fluxos apropriados para a atenção à essa população.

Em relação profissionais da área médica, estes achados devem ser considerados uma vez que corroboram a necessidade de avaliação microbiológica das úlceras venosas de difícil processo de cicatrização, para instituição da terapêutica adequada. Embora pareça um aspecto trivial, os resultados também sinalizam para a importância do compromisso com o zelo na indicação de antimicrobianos, monitoramento de seus efeitos, e 
supervisão da adesão do paciente, de modo a diminuir ao máximo as possibilidades de resistência microbiana

\section{REFERÊNCIAS}

1. Greer N, Foman N, Dorrian J, Fitzgerald P, MacDonald R, Rutks I et al. Advanced Wound Care Therapies for Non-Healing Diabetic, Venous, and Arterial Ulcers: A Systematic Review [Internet]. Washington: Department of Veterans Affairs; 2012 [acesso em: 30 jun 2014]. Disponível em: http://www.ncbi.nlm.nih.gov/pubmedhealth/PMH0054957/. VAESP Project \#09-009.

2. Cooper RA, Ameen H, Price P, McCulloch DA, Harding KG. A clinical investigation into the microbiological status of 'locally infected' leg ulcers. Int Wound ] [Internet]. 2009 [acesso em: 30 jun 2014];6(6):453-62. Disponível em:

http://dx.doi.org/10.1111/j.1742-481X.2009.00640.x. 3. Martins MA, Tipple AFV, Reis C, Santiago SB, Bachion MM. Úlcera crônica de perna de pacientes em tratamento ambulatorial: análise microbiológica e de suscetibilidade antimicrobiana. Ciênc. cuid. saúde. [Internet]. 2010 [acesso em: 30 jun 2014];9(3):464-70. Disponível em:

http://dx.doi.org/10.4025/cienccuidsaude.v9i3.8178.

4. Kali A, Srirangaraj S, Kumar S, Divya HA, Kalyani A, Umadevi S. Detection of metallo-beta-lactamase producing Pseudomonas aeruginosa in intensive care units. Australas Med J [Internet]. 2013 [acesso em: 30 jun 2014];6(12):686-93. Disponível em: http://dx.doi.org/10.4066/AMJ.2013.1824.

5. Rossi F, Andreazzi DB. Resistência bacteriana: interpretando o antibiograma. São Paulo: Editora Atheneu; 2005.

6. Villegas MV, Blanco MG, Sifuentes-Osornio J, Rossi F. Increasing prevalence of extended-spectrum-betalactamase among Gram-negative bacilli in Latin America - 2008 update from the Study for Monitoring Antimicrobial Resistance Trends (SMART). Braz J Infect Dis [Internet]. 2011 [acesso em: 30 jun 2014];15(1):34-9. Disponível em:

http://dx.doi.org/10.1590/S1413-86702011000100007.

7. Agência Nacional de Vigilância Sanitária. Nota Técnica No $1 / 2010$. Medidas para identificação, prevenção e controle de infecções relacionadas à assistência à saúde por microrganismos multirresistentes [Internet]. Brasília: ANVISA; 2010 [acesso em: 30 jun 2014]. Disponível em:

http://portal.anvisa.gov.br/wps/wcm/connect/6c8f7b804745781 1857ed53fbc4c6735/nota25-10-2010.pdf?MOD=AJPERES.

8. Clinical and Laboratory Standards Institute. Performance standards for antimicrobial susceptibility testing. Nineteenth informational supplement M100-S19. Wayne: Clinical and Laboratory Standards Institute; 2009.

9. Oplustil CP, Zocolli CM, Tobouti NR, Sinto SI. Procedimentos básicos em microbiologia clínica. $2^{a}$ ed. São Paulo: Sarvier; 2004.

10. Koneman, EW, Woods GL, Procop GW, Schreckenberger PC, Allen SD, Janda WM. Diagnóstico microbiológico: texto e atlas colorido. 7a ed. Rio de Janeiro: Medsi; 2008.

11. Jacoby GA. AmpC $\beta$-Lactamases. Clin Microbiol Rev [Internet]. 2009 [acesso em: 30 jun 2014];22(1):161-82. Disponível em: http://dx.doi.org/10.1128/CMR.00036-08. 12. Arakawa Y, Shibata N, Shibayama K, Kurokawa H, Yagi T, Fujiwara $\mathrm{H}$ et al. Convenient Test for Screening Metallo- $\beta$ Lactamase-Producing Gram-Negative Bacteria by Using Thiol Compounds. ] Clin Microbiol [Internet]. 2000 [acesso em: 30 jun 2014];38(1):40-3. Disponível em: http://jcm.asm.org/content/38/1/40.long.

13. Jazani $\mathrm{NH}$, Zahedi A, Garebagi N. Phenotypic detection of metallo- $\beta$-lactamase producing Pseudomonas aeruginosa isolated from Urmia hospitals. African Journal of Microbiology Research [Internet]. 2012 [acesso em: 30 jun 2014];6(7):1387-92. Disponível em: http://www.academicjournals.org/journal/AJMR/articleabstract/2A016B213762.

14. Gales AC, Menezes LC, Silbert S, Sader HS. Dissemination in distinct Brazilian regions of an epidemic carbapenem-resistant devido ao uso indiscriminado ou inadequado deste medicamento.
Pseudomonas aeruginosa producing SPM metallo-betalactamase. J Antimicrob Chemother [Internet]. 2003 [acesso em: 30 jun 2014];52(4):699-702. Disponível em: http://dx.doi.org/10.1093/jac/dkg416.

15. Murphy TA, Simm AM, Toleman MA, Jones RN, Walsh TR. Biochemical Characterization of the Acquired Metallo- $\beta$ Lactamase SPM-1 from Pseudomonas aeruginosa. Antimicrob Agents Chemother [Internet]. 2003 [acesso em: 30 jun 2014];47(2):582-7. Disponível em: http://dx.doi.org/10.1128/AAC.47.2.582-587.2003.

16. Leung GHY, Gray TJ; Cheong EYL, Haertsch P, Gottlieb T. Persistence of related bla-IMP-4 metallo-beta-lactamase producing Enterobacteriaceae from clinical and environmental specimens within a burns unit in Australia - a six-year retrospective study. Antimicrob Resist Infect Control [Internet]. 2013 [acesso em: 30 jun 2014];2:35. Disponível em: http://dx.doi.org/10.1186/2047-2994-2-35.

17. European Wound Management Association (EWMA). Position Document: Management of wound infection [Internet]. London: MEP Ltd; 2006 [acesso em: 30 jun 2014]. Disponível em: http://ewma.org/fileadmin/user_upload/EWMA/pdf/Position_Doc uments/2006/English_pos_doc_2006.pdf.

18. European Wound Management Association (EWMA). Position Document: Identifying criteria for wound infection [Internet]. London: MEP Ltd; 2005 [acesso em: 30 jun 2014]. Disponível em:

http://ewma.org/fileadmin/user_upload/EWMA/pdf/Position_Doc uments/2005_Wound_Infection_/English_pos_doc_final.pdf. 19. O'Meara S, Al-Kurdi D, Ologun Y, Ovington LG, Martyn-St JM, Richardson R. Antibiotics and antiseptics for venous leg ulcers. Cochrane Database Syst Rev [Internet]. 2014 [acesso em: 30 jun 2014]; Issue 1. Art. No.: CD003557. Disponível em: http://dx.doi.org/10.1002/14651858.CD003557.pub5. 20. Miller CN, Newall N, Kapp SE, Lewin G, Karimi L, Carville K et al. Wound Repair Regen [Internet]. 2010 [acesso em: 30 jun 2014];18(4):359-67. Disponível em:

http://dx.doi.org/10.1111/j.1524-475X.2010.00603.x.

21. Ferreira AM, Watanabe E, Nascimento AP, Andrade D, Ito IY Atividade antibacteriana in vitro de géis com diferentes concentrações de papaína. Rev. Eletr. Enf. [Internet]. 2008 [acesso em: 30 jun 2014];10(4):1035-40. Disponível em: http://www.fen.ufg.br/revista/v10/n4/v10n4a15.htm. 22. Levine NS, Lindberg RB, Mason Jr AD, Pruitt Jr BA. The quantitative swab culture and smear: A quick, simple method for determining the number of viable aerobic bacteria on open wounds. J Trauma. 1976 Feb;16(2):89-94.

23. Hernandez R. The use of systemic antibiotics in the treatment of chronic wounds. Dermatol Ther [Internet]. 2006 [acesso em: 30 jun 2014];19(6):326-37. Disponível em: http://dx.doi.org/10.1111/j.1529-8019.2006.00091.x. 24. Wolcott RD, Gontcharova V, Sun Y, Dowd SE. Evaluation of the bacterial diversity among and within individual venous leg ulcers using bacterial tag-encoded FLX and titanium amplicon pyrosequencing and metagenomic approaches. BMC Microbiol [Internet]. 2009 [acesso em: 30 jun 2014];9:226. Disponível em: http://dx.doi.org/10.1186/1471-2180-9-226.

25. Bali NK, Fomda BA, Bashir H, Zahoor D, Lone S, Koul PA. Emergence of carbapenem-resistant Acinetobacter in a temperate north Indian State. Br J Biomed Sci. 2013;70(4):15660.

Artigo recebido em 20/06/2013. Aprovado para publicação em 07/02/2014. Artigo publicado em 30/06/2014. 